\title{
Necessary and Sufficient Conditions for the Existence of a Positive Definite Solution for the Matrix Equation $X+\sum_{i=1}^{m} A_{i}^{T} X^{\delta_{i}} A_{i}=I$
}

\author{
Naglaa M. El-Shazly \\ Department of Mathematics, Facultyof Science, Menoufia University, Shebin El-Koom, Egypt
}

Correspondence should be addressed to Naglaa M. El-Shazly; naglaamoh1@yahoo.com

Received 19 December 2012; Revised 26 March 2013; Accepted 14 May 2013

Academic Editor: Theodore E. Simos

Copyright (c) 2013 Naglaa M. El-Shazly. This is an open access article distributed under the Creative Commons Attribution License, which permits unrestricted use, distribution, and reproduction in any medium, provided the original work is properly cited.

In this paper necessary and sufficient conditions for the matrix equation $X+\sum_{i=1}^{m} A_{i}^{T} X^{\delta_{i}} A_{i}=I$ to have a positive definite solution $X$ are derived, where $-1<\delta_{i}<0, I$ is an $n \times n$ identity matrix, $A_{i}$ are $n \times n$ nonsingular real matrices, and $m$ is an odd positive integer. These conditions are used to propose some properties on the matrices $A_{i}, i=1,2, \ldots, m$. Moreover, relations between the solution $X$ and the matrices $A_{i}$ are derived.

\section{Introduction}

Consider the nonlinear matrix equation

$$
X+\sum_{i=1}^{m} A_{i}^{T} X^{\delta_{i}} A_{i}=I, \quad-1<\delta_{i}<0,
$$

where $I$ is an $n \times n$ identity matrix, $A_{i}$ are $n \times n$ nonsingular real matrices, and $m$ is an odd positive integer $\left(A_{i}^{*}\right.$ stands for the conjugate transpose of the matrix $\left.A_{i}\right)$. Several authors $[1-$ 13 ] have studied the existence, the rate of convergence, as well as the necessary and sufficient conditions of the existence of positive definite solutions of similar kinds of nonlinear matrix equations.

This paper is organized as follows. First, in Section 2, we introduce some notations, lemmas, and theorems that will be needed to develop this work. In Section 3, we present necessary and sufficient conditions for the existence of a positive definite solution of (1). In Section 4, some applications of the obtained results as well as relations between the solution $X$ and the matrices $A_{i}$ are given.

\section{Preliminaries}

In this section, we present some notations, lemmas, and theorems that will be needed to develop this paper. We denote by $r(A), \lambda(A)$, and $\|A\|$ the spectral radius of $A$, the eigenvalues of $A$, and the Euclidean norm of $A$, respectively. The notation $A>B(A \geq B)$ indicates that $A-B$ is positive definite (semidefinite).

Lemma 1. Let $E$ and $F$ be two arbitrary compatible matrices. Then,

$$
r\left(E^{T} F-F^{T} E\right) \leq r\left(E^{T} E+F^{T} F\right) .
$$

The proof of this lemma is given in [14, Lemma 6].

Theorem 2 (Cholesky Decomposition). If $G$ is an $n \times n$ symmetric positive definite matrix, then there exists a triangular $n \times n$ matrix $M$ with positive diagonal entries such that $G=$ $M^{T} M$.

For proof, see [15, page 141].

Theorem 3 (C-S decomposition [15, page 77] and [16, page 37]). If $Q$ is an orthogonal matrix where $Q=\left[\begin{array}{ll}Q_{11} & Q_{12} \\ Q_{21} & Q_{22}\end{array}\right]$, then there exist orthogonal matrices $U_{1}, U_{2}, V_{1}, V_{2}$ and diagonal matrices $\Gamma \geq 0, \Sigma \geq 0$ such that

$$
Q=\left[\begin{array}{cc}
U_{1} & 0 \\
0 & U_{2}
\end{array}\right]\left[\begin{array}{cc}
\Gamma & -\Sigma \\
\Sigma & \Gamma
\end{array}\right]\left[\begin{array}{cc}
V_{1} & 0 \\
0 & V_{2}
\end{array}\right],
$$

where $\Gamma^{2}+\Sigma^{2}=I$. 


\section{Necessary and Sufficient Conditions}

In this section, we derive both necessary and sufficient conditions for the existence of a positive definite solution of the nonlinear matrix equation (1).

Theorem 4. The matrix equation (1) has a solution $X$ (symmetric and positive definite) if and only if $A_{i}, i=1,2, \ldots, m$, admit the following factorization:

$$
\sum_{i=1}^{m} A_{i}=\sum_{i=1}^{m}\left(W^{T} W\right)^{-\delta_{i} / 2} Z_{i}
$$

where $W$ is a nonsingular square matrix and the columns of

$$
\left(\begin{array}{c}
W \\
Z_{1} \\
Z_{2} \\
\vdots \\
Z_{m}
\end{array}\right)
$$

are orthonormal. In this case, we take $X=W^{T} W$ as a solution of the matrix equation (1).

Proof. If (1) has a solution $X$, then one can write $X$ as $X=$ $W^{T} W$ for some nonsingular matrix $W$. In particular, $W$ can be chosen to be triangular using Theorem 2; thus, we have

$$
\begin{aligned}
X & +\sum_{i=1}^{m} A_{i}^{T} X^{\delta_{i}} A_{i} \\
& =W^{T} W+\sum_{i=1}^{m} A_{i}^{T}\left(W^{T} W\right)^{\delta_{i}} A_{i} \\
& =W^{T} W+\sum_{i=1}^{m} A_{i}^{T}\left(W^{T} W\right)^{\delta_{i} / 2}\left(W^{T} W\right)^{\delta_{i} / 2} A_{i} .
\end{aligned}
$$

Then (1) can be rewritten as

$$
W^{T} W+\sum_{i=1}^{m}\left(\left(W^{T} W\right)^{\delta_{i} / 2} A_{i}\right)^{T}\left(\left(W^{T} W\right)^{\delta_{i} / 2} A_{i}\right)=I,
$$

or equivalently

$$
\left[W^{T}, \sum_{i=1}^{m}\left(\left(W^{T} W\right)^{\delta_{i} / 2} A_{i}\right)^{T}\right]\left[\sum_{i=1}^{m}\left(\left(W^{T} W\right)^{\delta_{i} / 2} A_{i}\right)\right]=I .
$$

Let

$$
\sum_{i=1}^{m}\left(\left(W^{T} W\right)^{\delta_{i} / 2} A_{i}\right)=\sum_{i=1}^{m} Z_{i}
$$

Then

$$
\sum_{i=1}^{m} A_{i}=\sum_{i=1}^{m}\left(W^{T} W\right)^{-\delta_{i} / 2} Z_{i}
$$

and (8) means that the columns of

$$
\left(\begin{array}{c}
W \\
Z_{1} \\
Z_{2} \\
\vdots \\
Z_{m}
\end{array}\right)
$$

are orthonormal.

Conversely, suppose that $A_{i}, i=1,2, \ldots, m$ have the decomposition (4). Set $X=W^{T} W$. Then

$$
\begin{gathered}
X+\sum_{i=1}^{m} A_{i}^{T} X^{\delta_{i}} A_{i} \\
=W^{T} W+\sum_{i=1}^{m}\left(\left(W^{T} W\right)^{-\delta_{i} / 2} Z_{i}\right)^{T} \\
\times\left(W^{T} W\right)^{\delta_{i}}\left(\left(W^{T} W\right)^{-\delta_{i} / 2} Z_{i}\right) \\
=W^{T} W+\sum_{i=1}^{m}\left(Z_{i}^{T}\left(W^{T} W\right)^{-\delta_{i} / 2}\right) \\
\times\left(W^{T} W\right)^{\delta_{i} / 2}\left(W^{T} W\right)^{\delta_{i} / 2} \\
\times\left(\left(W^{T} W\right)^{-\delta_{i} / 2} Z_{i}\right) \\
=W^{T} W+\sum_{i=1}^{m} Z_{i}^{T} Z_{i}=I .
\end{gathered}
$$

That is, $X$ is a solution to the matrix equation (1).

Theorem 5. The matrix equation (1) has a solution if and only if there exist orthogonal matrices $P$ and $U_{2}$ and diagonal matrices $\Gamma>0$ and $\Sigma \geq 0$ with $((m+1) / 2)\left(\Gamma^{2}+\Sigma^{2}\right)=I$ such that

$$
\begin{aligned}
\sum_{i=1}^{m} A_{i}= & \sum_{(i \text { odd })}^{m}\left(P^{T} \Gamma^{2} P\right)^{-\delta_{i} / 2} U_{i+1} \Sigma P \\
& +\sum_{(i \text { even })}^{m-1}\left(P^{T} \Gamma^{2} P\right)^{-\delta_{i} / 2} U_{i+1} \Gamma P .
\end{aligned}
$$

In this case, $X=P^{T} \Gamma^{2} P$ is a solution of (1).

Proof. Suppose that the matrix equation (1) has a solution. Then from Theorem $4, A_{i}, i=1,2, \ldots, m$, have the form

$$
\sum_{i=1}^{m} A_{i}=\sum_{i=1}^{m}\left(W^{T} W\right)^{-\delta_{i} / 2} Z_{i}
$$

Since

$$
\left(\begin{array}{c}
W \\
Z_{1} \\
Z_{2} \\
\vdots \\
Z_{m}
\end{array}\right)
$$


is column-orthonormal, it can be extended to an orthogonal matrix

$$
\left(\begin{array}{cc}
W & U \\
Z_{1} & V_{1} \\
Z_{2} & V_{2} \\
\vdots & \vdots \\
Z_{m} & V_{m}
\end{array}\right)
$$

and applying Theorem 3, there exist orthogonal matrices $U_{1}, U_{2}, \ldots, U_{m+1}, P, Q$ and diagonal matrices $\Gamma>0$ and $\Sigma \geq 0$ such that

$$
\left(\begin{array}{cc}
W & U \\
Z_{1} & V_{1} \\
Z_{2} & V_{2} \\
\vdots & \vdots \\
Z_{m} & V_{m}
\end{array}\right)=\left(\begin{array}{cc}
U_{1} & 0 \\
0 & U_{2} \\
\vdots & \vdots \\
U_{m} & 0 \\
0 & U_{m+1}
\end{array}\right)\left(\begin{array}{cc}
\Gamma & -\Sigma \\
\Sigma & \Gamma
\end{array}\right)\left(\begin{array}{ll}
P & 0 \\
0 & Q
\end{array}\right)
$$

where $((m+1) / 2)\left(\Gamma^{2}+\Sigma^{2}\right)=I$.

This decomposition is a generalization for Theorem 3 in [15, page 77] and [16, page 37]. It is clear that if we set $m=1$, we get the special case of that theorem.

Thus $W=U_{1} \Gamma P$, and

$$
\sum_{i=1}^{m} Z_{i}=\sum_{i=1,3}^{m} U_{i+1} \Sigma P+\sum_{i=2,4}^{m-1} U_{i+1} \Gamma P .
$$

Then (4) can be rewritten as follows:

$$
\begin{aligned}
\sum_{i=1}^{m} A_{i}= & \sum_{i=1}^{m}\left(W^{T} W\right)^{-\delta_{i} / 2} Z_{i} \\
= & \sum_{i=1,3}^{m}\left(P^{T} \Gamma^{2} P\right)^{-\delta_{i} / 2} U_{i+1} \Sigma P \\
& +\sum_{i=2,4}^{m-1}\left(P^{T} \Gamma^{2} P\right)^{-\delta_{i} / 2} U_{i+1} \Gamma P .
\end{aligned}
$$

Conversely, suppose that $A_{i}, i=1,2, \ldots, m$ have the decomposition (13). Let $X=P^{T} \Gamma^{2} P$. Then

$$
\begin{aligned}
X+\sum_{i=1}^{m} A_{i}^{T} X^{\delta_{i}} A_{i}= & \left(U_{1} \Gamma P\right)^{T}\left(U_{1} \Gamma P\right) \\
& +\sum_{i=1,3}^{m}\left(\left(P^{T} \Gamma^{2} P\right)^{-\delta_{i} / 2} U_{i+1} \Sigma P\right)^{T}\left(P^{T} \Gamma^{2} P\right)^{\delta_{i}} \\
& \times\left(\left(P^{T} \Gamma^{2} P\right)^{-\delta_{i} / 2} U_{i+1} \Sigma P\right) \\
& +\sum_{i=2,4}^{m-1}\left(\left(P^{T} \Gamma^{2} P\right)^{-\delta_{i} / 2} U_{i+1} \Gamma P\right)^{T}\left(P^{T} \Gamma^{2} P\right)^{\delta_{i}} \\
& \times\left(\left(P^{T} \Gamma^{2} P\right)^{-\delta_{i} / 2} U_{i+1} \Gamma P\right) \\
= & P^{T} \Gamma^{2} P+\left(\left(P^{T} \Gamma^{2} P\right)^{-\delta_{1} / 2} U_{2} \Sigma P\right)^{T}
\end{aligned}
$$

$$
\begin{aligned}
& \times\left(P^{T} \Gamma^{2} P\right)^{\delta_{1}}\left(\left(P^{T} \Gamma^{2} P\right)^{-\delta_{1} / 2} U_{2} \Sigma P\right) \\
& +\left(\left(P^{T} \Gamma^{2} P\right)^{-\delta_{3} / 2} U_{4} \Sigma P\right)^{T}\left(P^{T} \Gamma^{2} P\right)^{\delta_{3}} \\
& \times\left(\left(P^{T} \Gamma^{2} P\right)^{-\delta_{3} / 2} U_{4} \Sigma P\right) \\
& +\cdots+\left(\left(P^{T} \Gamma^{2} P\right)^{-\delta_{m} / 2} U_{m+1} \Sigma P\right)^{T} \\
& \times\left(P^{T} \Gamma^{2} P\right)^{\delta_{m}}\left(\left(P^{T} \Gamma^{2} P\right)^{-\delta_{m} / 2} U_{m+1} \Sigma P\right) \\
& +\left(\left(P^{T} \Gamma^{2} P\right)^{-\delta_{2} / 2} U_{3} \Gamma P\right)^{T}\left(P^{T} \Gamma^{2} P\right)^{\delta_{2}} \\
& \times\left(\left(P^{T} \Gamma^{2} P\right)^{-\delta_{2} / 2} U_{3} \Gamma P\right) \\
& +\cdots+\left(\left(P^{T} \Gamma^{2} P\right)^{-\delta_{m-1} / 2} U_{m} \Gamma P\right)^{T} \\
& \times\left(P^{T} \Gamma^{2} P\right)^{\delta_{m-1}}\left(\left(P^{T} \Gamma^{2} P\right)^{-\delta_{m-1} / 2} U_{m} \Gamma P\right) \\
& =P^{T} \Gamma^{2} P+\left(P^{T} \Sigma U_{2}^{T}\right)\left(P^{T} \Gamma^{2} P\right)^{-\delta_{1} / 2} \\
& \times\left(P^{T} \Gamma^{2} P\right)^{\delta_{1}}\left(P^{T} \Gamma^{2} P\right)^{-\delta_{1} / 2} U_{2} \Sigma P \\
& +\left(P^{T} \Sigma U_{4}^{T}\right)\left(P^{T} \Gamma^{2} P\right)^{-\delta_{3} / 2} \\
& \times\left(P^{T} \Gamma^{2} P\right)^{\delta_{3}}\left(P^{T} \Gamma^{2} P\right)^{-\delta_{3} / 2} U_{4} \Sigma P \\
& +\ldots+\left(P^{T} \Sigma U_{m+1}^{T}\right)\left(P^{T} \Gamma^{2} P\right)^{-\delta_{m} / 2} \\
& \times\left(P^{T} \Gamma^{2} P\right)^{\delta_{m}}\left(P^{T} \Gamma^{2} P\right)^{-\delta_{m} / 2} U_{m+1} \Sigma P \\
& +\left(P^{T} \Gamma U_{3}^{T}\right)\left(P^{T} \Gamma^{2} P\right)^{-\delta_{2} / 2} \\
& \times\left(P^{T} \Gamma^{2} P\right)^{\delta_{2}}\left(P^{T} \Gamma^{2} P\right)^{-\delta_{2} / 2} U_{3} \Gamma P \\
& +\left(P^{T} \Gamma U_{m}^{T}\right)\left(P^{T} \Gamma^{2} P\right)^{-\delta_{m-1} / 2} \\
& \times\left(P^{T} \Gamma^{2} P\right)^{\delta_{m-1}}\left(P^{T} \Gamma^{2} P\right)^{-\delta_{m-1} / 2} U_{m} \Gamma P \\
& =P^{T} \Gamma^{2} P+P^{T} \Sigma U_{2}^{T} U_{2} \Sigma P+P^{T} \Sigma U_{4}^{T} U_{4} \Sigma P \\
& +\cdots+P^{T} \Sigma U_{m+1}^{T} U_{m+1} \Sigma P \\
& +P^{T} \Gamma U_{3}^{T} U_{3} \Gamma P+\cdots+P^{T} \Gamma U_{m}^{T} U_{m} \Gamma P \\
& =P^{T} \Gamma^{2} P+P^{T} \Sigma^{2} P+P^{T} \Sigma^{2} P \\
& +\cdots+P^{T} \Sigma^{2} P+P^{T} \Gamma^{2} P+\cdots+P^{T} \Gamma^{2} P \\
& =P^{T}\left(\frac{m+1}{2}\left(\Gamma^{2}+\Sigma^{2}\right)\right) P=P^{T} P=\mathrm{I} \text {. }
\end{aligned}
$$

This shows that $X$ is a solution to the matrix equation (1). 


\section{Main Results}

In this section, we explain some properties of (1) and we obtain relations between the solution $X$ and the matrices $A_{i}$.

Theorem 6. If the matrix equation (1) has a positive definite solution $X$, then $\left\|\sum_{i=1}^{m} A_{i}\right\|<m$.

Proof. Suppose that (1) has a solution. Then by Theorem 5, $\sum_{i=1}^{m} A_{i}$ has the decomposition (13).

Then

$$
\begin{aligned}
\left\|\sum_{i=1}^{m} A_{i}\right\| & \| \sum_{i=1,3}^{m}\left(P^{T} \Gamma^{2} P\right)^{-\delta_{i} / 2} U_{i+1} \Sigma P \\
& +\sum_{i=2,4}^{m-1}\left(P^{T} \Gamma^{2} P\right)^{-\delta_{i} / 2} U_{i+1} \Gamma P \| \\
\leq & \left\|\sum_{i=1,3}^{m}\left(P^{T} \Gamma^{2} P\right)^{-\delta_{i} / 2} U_{i+1} \Sigma P\right\| \\
& +\left\|\sum_{i=2,4}^{m-1}\left(P^{T} \Gamma^{2} P\right)^{-\delta_{i} / 2} U_{i+1} \Gamma P\right\| \\
\leq & \sum_{i=1,3}^{m}\left\|\left(P^{T} \Gamma^{2} P\right)^{-\delta_{i} / 2}\right\|\left\|U_{i+1}\right\|\|\Sigma\|\|P\| \\
& +\sum_{i=2,4}^{m-1}\left\|\left(P^{T} \Gamma^{2} P\right)^{-\delta_{i} / 2}\right\|\left\|U_{i+1}\right\|\|\Gamma\|\|P\| .
\end{aligned}
$$

Since each $U_{i+1}, i=1,2, \ldots, m$, is orthogonal, also $P$ is orthogonal. So

$$
\begin{gathered}
\left\|\sum_{i=1}^{m} A_{i}\right\| \\
\leq \sum_{i=1,3}^{m}\left\|\left(P^{T} \Gamma^{2} P\right)^{-\delta_{i} / 2}\right\|\|\Sigma\| \\
+\sum_{i=2,4}^{m-1}\left\|\left(P^{T} \Gamma^{2} P\right)^{-\delta_{i} / 2}\right\|\|\Gamma\| \\
\leq \sum_{i=1,3}^{m}\left\|P^{T} \Gamma^{2} P\right\|^{-\delta_{i} / 2}\|\Sigma\| \\
+\sum_{i=2,4}^{m-1}\left\|P^{T} \Gamma^{2} P\right\|^{-\delta_{i} / 2}\|\Gamma\|
\end{gathered}
$$

$$
\begin{aligned}
& =\sum_{i=1,3}^{m}\left\|\Gamma^{2}\right\|^{-\delta_{i} / 2}\|\Sigma\|+\sum_{i=2,4}^{m-1}\left\|\Gamma^{2}\right\|^{-\delta_{i} / 2}\|\Gamma\| \\
& \leq \sum_{i=1,3}^{m}\|\Gamma\|^{-\delta_{i}}\|\Sigma\|+\sum_{i=2,4}^{m-1}\|\Gamma\|^{-\delta_{i}}\|\Gamma\| .
\end{aligned}
$$

Since we choose $-1<\delta_{i}<0$, then we have $\|\Gamma\|^{-\delta_{i}} \leq\|\Gamma\|<1$, and therefore

$$
\sum_{i=1,3}^{m}\|\Gamma\|^{-\delta_{i}} \leq\left(\frac{m+1}{2}\right), \quad \sum_{i=2,4}^{m-1}\|\Gamma\|^{-\delta_{i}} \leq\left(\frac{m-1}{2}\right) .
$$

Thus,

$$
\begin{aligned}
\left\|\sum_{i=1}^{m} A_{i}\right\| \leq & \left(\frac{m+1}{2}\right)\left(\sqrt{\frac{2}{m+1}}\right) \\
& +\left(\frac{m-1}{2}\right)\left(\sqrt{\frac{2}{m+1}}\right)=m \sqrt{\frac{2}{m+1}}<m .
\end{aligned}
$$

The last inequality follows from the fact $((m+1) / 2)\left(\Gamma^{2}+\Sigma^{2}\right)=$ $I$, which yields that $\|\Gamma\| \leq \sqrt{2 /(m+1)}$ and $\|\Sigma\|<\sqrt{2 /(m+1)}$.

In the following theorem, using the necessary and sufficient conditions which we have derived, we state and prove some inequalities in case of the matrix equation (1) having positive definite solution.

Theorem 7. Suppose that the matrix equation (1) has a positive definite solution $X$; then the following hold:

(i) $\sum_{i=1}^{m} X^{-\delta_{i}}>\sum_{i=1}^{m} A_{i} A_{i}^{T}$,

(ii) $I-\sum_{i=1}^{m} A_{i}^{T} X^{\delta_{i}+1} A_{i}-\sum_{i=1}^{m} X^{\left(\delta_{i}+1\right) / 2} A_{i} A_{i}^{T} X^{\left(\delta_{i}+1\right) / 2}>0$,

(iii) $r\left(\sum_{i=1}^{m} A_{i}\right) \leq(1 / 2) \sqrt{2 /(m+1)}((3 m-1) /(m+1))$,

(iv) $r\left(\sum_{i=1}^{m} X^{\left(1+\delta_{i}\right) / 2} A_{i}-\sum_{i=1}^{m} A_{i}^{T} X^{\left(1+\delta_{i}\right) / 2}\right) \leq 1$,

(v) $r\left(\sum_{i=1}^{m} X^{\left(1+\delta_{i}\right) / 2} A_{i}+\sum_{i=1}^{m} A_{i}^{T} X^{\left(1+\delta_{i}\right) / 2}\right) \leq 1$.

Proof. (i) By Theorem 4, we have $X=W^{T} W$ and $\sum_{i=1}^{m} A_{i}=$ $\sum_{i=1}^{m}\left(W^{T} W\right)^{-\delta_{i} / 2} Z_{i}$. Then

$$
\begin{aligned}
\sum_{i=1}^{m} X^{-\delta_{i}}-\sum_{i=1}^{m} A_{i} A_{i}^{T} \\
=\sum_{i=1}^{m}\left(W^{T} W\right)^{-\delta_{i}} \\
\quad-\sum_{i=1}^{m}\left(W^{T} W\right)^{-\delta_{i} / 2} Z_{i} Z_{i}^{T}\left(W^{T} W\right)^{-\delta_{i} / 2}
\end{aligned}
$$




$$
\begin{aligned}
= & \sum_{i=1}^{m}\left(W^{T} W\right)^{-\delta_{i} / 2}\left(W^{T} W\right)^{-\delta_{i} / 2} \\
& -\sum_{i=1}^{m}\left(W^{T} W\right)^{-\delta_{i} / 2} Z_{i} Z_{i}^{T}\left(W^{T} W\right)^{-\delta_{i} / 2} \\
= & \sum_{i=1}^{m}\left(W^{T} W\right)^{-\delta_{i} / 2}\left[I-\sum_{i=1}^{m} Z_{i} Z_{i}^{T}\right]\left(W^{T} W\right)^{-\delta_{i} / 2}>0,
\end{aligned}
$$

since $\lambda\left(Z_{i} Z_{i}^{T}\right)=\lambda\left(Z_{i}^{T} Z_{i}\right), i=1,2, \ldots, m$, and $I-\sum_{i=1}^{m} Z_{i}^{T} Z_{i}=$ $W^{T} W>0$, and, hence, $I-\sum_{i=1}^{m} Z_{i} Z_{i}^{T}>0$. Thus, part (i) is proved.

(ii) Replacing $I$ by $W^{T} W+\sum_{i=1}^{m} Z_{i}^{T} Z_{i}$, we get

$$
\begin{aligned}
I- & \sum_{i=1}^{m} A_{i}^{T} X^{\delta_{i}+1} A_{i}-\sum_{i=1}^{m} X^{\left(\delta_{i}+1\right) / 2} A_{i} A_{i}^{T} X^{\left(\delta_{i}+1\right) / 2} \\
= & W^{T} W+\sum_{i=1}^{m} Z_{i}^{T} Z_{i}-\sum_{i=1}^{m} A_{i}^{T} X^{\delta_{i}+1} A_{i} \\
& -\sum_{i=1}^{m} X^{\left(\delta_{i}+1\right) / 2} A_{i} A_{i}^{T} X^{\left(\delta_{i}+1\right) / 2} \\
= & W^{T} W+\sum_{i=1}^{m} Z_{i}^{T} Z_{i}-\sum_{i=1}^{m}\left(\left(W^{T} W\right)^{-\delta_{i} / 2} Z_{i}\right)^{T} \\
& -\sum_{i=1}^{m}\left(W^{T} W\right)^{\left(\delta_{i}+1\right) / 2}\left(\left(W^{T} W\right)^{-\delta_{i} / 2} Z_{i}\right) \\
& \times\left(\left(W^{T} W\right)^{-\delta_{i} / 2} Z_{i}\right)^{T}\left(W^{T} W\right)^{\left(\delta_{i}+1\right) / 2} \\
= & W^{T} W+\sum_{i=1}^{m} Z_{i}^{T} Z_{i} \\
& =\left(W_{i=1}^{m} Z_{i}^{T}\left(W^{T} W\right)^{T / 2}\left(W^{T} W\right)^{-\delta_{i} / 2} Z_{i}\right) \\
& -\sum_{i=1}^{m} Z_{i}^{T}\left(W^{T} W\right)^{-\delta_{i} / 2}+\left(\sum_{i=1}^{m} Z_{i}^{T} Z_{i}\right. \\
& \times\left(Z_{i}^{T}\left(W^{T} W\right)^{-\delta_{i} / 2}\right)\left(W^{T} W\right)^{\left(\delta_{i}+1\right) / 2} \sum_{i=1}^{m} Z_{i} Z_{i}^{T}\left(W^{T} W\right)^{1 / 2} \\
& \quad-\sum_{i=1}^{m}\left(W^{T} W\right)^{\left(\delta_{i}+1\right) / 2}\left(\left(W^{T} W\right)^{-\delta_{i} / 2} Z_{i}\right) \\
& \times\left(W^{T} W\right)^{\delta_{i}+1}\left(W^{T} W\right)^{-\delta_{i} / 2} Z_{i}
\end{aligned}
$$

$$
\begin{aligned}
= & \left(W^{T} W\right)^{1 / 2}\left(I-\sum_{i=1}^{m} Z_{i} Z_{i}^{T}\right) \\
& \times\left(W^{T} W\right)^{1 / 2}+\sum_{i=1}^{m} Z_{i}^{T}\left(I-W^{T} W\right) Z_{i}>0 .
\end{aligned}
$$

This completes the proof of (ii).

(iii) Using Theorem 6, we get

$$
\begin{aligned}
\lambda\left(\sum_{i=1}^{m} A_{i}\right)=\lambda( & \sum_{i=1,3}^{m}\left(P^{T} \Gamma^{2} P\right)^{-\delta_{i} / 2} U_{i+1} \Sigma P \\
& \left.+\sum_{i=2,4}^{m-1}\left(P^{T} \Gamma^{2} P\right)^{-\delta_{i} / 2} U_{i+1} \Gamma P\right),
\end{aligned}
$$

$r\left(\sum_{i=1}^{m} A_{i}\right)$

$$
=\max _{i} \mid \lambda\left(\sum_{i=1,3}^{m}\left(P^{T} \Gamma^{2} P\right)^{-\delta_{i} / 2} U_{i+1} \Sigma P\right.
$$$$
\left.+\sum_{i=2,4}^{m-1}\left(P^{T} \Gamma^{2} P\right)^{-\delta_{i} / 2} U_{i+1} \Gamma P\right) \mid
$$$$
\leq \|\left(\sum_{i=1,3}^{m}\left(P^{T} \Gamma^{2} P\right)^{-\delta_{i} / 2} U_{i+1} \Sigma P\right.
$$$$
\left.+\sum_{i=2,4}^{m-1}\left(P^{T} \Gamma^{2} P\right)^{-\delta_{i} / 2} U_{i+1} \Gamma P\right) \|
$$

$$
\leq\left\|\sum_{i=1,3}^{m}\left(P^{T} \Gamma^{2} P\right)^{-\delta_{i} / 2} U_{i+1} \Sigma P\right\|
$$$$
+\left\|\sum_{i=2,4}^{m-1}\left(P^{T} \Gamma^{2} P\right)^{-\delta_{i} / 2} U_{i+1} \Gamma P\right\|
$$$$
=\sum_{i=1,3}^{m}\left\|\left(P^{T} \Gamma^{2} P\right)^{-\delta_{i} / 2} \Sigma\right\|
$$

$$
+\sum_{i=2,4}^{m-1}\left\|\left(P^{T} \Gamma^{2} P\right)^{-\delta_{i} / 2} \Gamma\right\| .
$$

Since we choose $-1<\delta_{i}<0$, then we have $0<\left(-\delta_{i}\right) / 2<$ $1 / 2<1$, and therefore $\left(P^{T} \Gamma^{2} P\right)^{-\delta_{i} / 2} \leq\left(P^{T} \Gamma^{2} P\right), i=$ $1,2, \ldots, m$; then

$$
\begin{aligned}
r\left(\sum_{i=1}^{m} A_{i}\right) \leq & \frac{m+1}{2}\left\|\left(P^{T} \Gamma^{2} P\right) \Sigma\right\| \\
& +\frac{m-1}{2}\left\|\left(P^{T} \Gamma^{2} P\right) \Gamma\right\| \\
= & \frac{m+1}{2}\left\|P^{T} \Sigma \Gamma^{2} P\right\|+\frac{m-1}{2}\left\|P^{T} \Gamma^{3} P\right\|
\end{aligned}
$$




$$
\begin{aligned}
& =\frac{m+1}{2}\left\|\Sigma \Gamma^{2}\right\|+\frac{m-1}{2}\left\|\Gamma^{3}\right\| \\
& \leq \frac{m+1}{2}\|\Sigma \Gamma \Gamma\|+\frac{m-1}{2}\|\Gamma\|^{3} \\
& \leq \frac{m+1}{2}\|\Sigma \Gamma\|\|\Gamma\|+\frac{m-1}{2}\|\Gamma\|^{3} .
\end{aligned}
$$

Let $\Sigma=\operatorname{diag}\left(\sigma_{j}\right), \Gamma=\operatorname{diag}\left(\gamma_{j}\right)$. Then $((m+1) / 2)\left(\sigma_{j}^{2}+\gamma_{j}^{2}\right)=1$. Thus

$$
\begin{aligned}
& r\left(\sum_{i=1}^{m} A_{i}\right) \\
& \leq \frac{m+1}{2} \sqrt{\frac{2}{m+1}}\|\Sigma \Gamma\|+\frac{m-1}{2}\left(\sqrt{\frac{2}{m+1}}\right)^{3} \\
& =\frac{m+1}{2} \sqrt{\frac{2}{m+1}} \max _{j}\left\{\sigma_{j} \gamma_{j}\right\}+\frac{m-1}{2}\left(\sqrt{\frac{2}{m+1}}\right)^{3} \\
& \leq \frac{m+1}{2} \sqrt{\frac{2}{m+1}} \max _{j} \frac{\sigma_{j}^{2}+\gamma_{j}^{2}}{2}+\frac{m-1}{2}\left(\sqrt{\frac{2}{m+1}}\right)^{3} \\
& =\frac{1}{2} \sqrt{\frac{2}{m+1}}\left(\frac{3 m-1}{m+1}\right) .
\end{aligned}
$$

(iv) Appling Lemma 1, we get

$$
\begin{gathered}
r\left(\sum_{i=1}^{m} X^{\left(1+\delta_{i}\right) / 2} A_{i}-\sum_{i=1}^{m} A_{i}^{T} X^{\left(1+\delta_{i}\right) / 2}\right) \\
=r\left(\sum_{i=1}^{m}\left(W^{T} W\right)^{\left(1+\delta_{i}\right) / 2}\left(W^{T} W\right)^{-\delta_{i} / 2} Z_{i}\right. \\
\left.-\sum_{i=1}^{m} Z_{i}^{T}\left(W^{T} W\right)^{-\delta_{i} / 2}\left(W^{T} W\right)^{\left(1+\delta_{i}\right) / 2}\right) \\
=r\left(\sum_{i=1}^{m}\left(W^{T} W\right)^{1 / 2} Z_{i}-\sum_{i=1}^{m} Z_{i}^{T}\left(W^{T} W\right)^{1 / 2}\right) \\
\leq r\left(\left(W^{T} W\right)+\sum_{i=1}^{m} Z_{i}^{T} Z_{i}\right)=r(I)=1 .
\end{gathered}
$$

(v) Consider

$$
\begin{gathered}
I \pm\left(\sum_{i=1}^{m} X^{\left(1+\delta_{i}\right) / 2} A_{i}+\sum_{i=1}^{m} A_{i}^{T} X^{\left(1+\delta_{i}\right) / 2}\right) \\
=W^{T} W+\sum_{i=1}^{m} Z_{i}^{T} Z_{i}
\end{gathered}
$$

$$
\begin{aligned}
& \pm\left(\sum_{i=1}^{m}\left(W^{T} W\right)^{1 / 2} Z_{i}+\sum_{i=1}^{m} Z_{i}^{T}\left(W^{T} W\right)^{1 / 2}\right) \\
& =\left(\left(W^{T} W\right)^{1 / 2} \pm \sum_{i=1}^{m} Z_{i}\right)^{T}\left(\left(W^{T} W\right)^{1 / 2} \pm \sum_{i=1}^{m} Z_{i}\right) \geq 0 .
\end{aligned}
$$

Therefore, $r\left(\sum_{i=1}^{m} X^{\left(1+\delta_{i}\right) / 2} A_{i}+\sum_{i=1}^{m} A_{i}^{T} X^{\left(1+\delta_{i}\right) / 2}\right) \leq 1$.

\section{Conclusion}

In this paper, both necessary and sufficient conditions for the nonlinear matrix equation $X+\sum_{i=1}^{m} A_{i}^{T} X^{\delta_{i}} A_{i}=I$ to have a positive definite solution $X$ are given, where $-1<$ $\delta_{i}<0, A_{i}$ are $n \times n$ nonsingular real matrices, and $m$ is an odd positive integer. Some properties of this matrix equation are explained. Also, relations between the positive definite solution $X$ and the matrices $A_{i}, i=1,2, \ldots, m$, are presented. ffk

\section{References}

[1] X. Duan, A. Liao, and B. Tang, "On the nonlinear matrix equation $X-\sum_{i=1}^{m} A_{i}^{*} X^{\delta_{i}} A_{i}=Q$," Linear Algebra and its Applications, vol. 429, no. 1, pp. 110-121, 2008.

[2] S. M. El-Sayed and M. A. Ramadan, "On the existence of a positive definite solution of the matrix equation $X-A^{*} \sqrt[2 m]{X^{-1}} A=$ I," International Journal of Computer Mathematics, vol. 76, no. 3, pp. 331-338, 2001.

[3] J. C. Engwerda, A. C. M. Ran, and A. L. Rijkeboer, "Necessary and sufficient conditions for the existence of a positive definite solution of the matrix equation $X+A^{*} X^{-1} A=Q$," Linear Algebra and its Applications, vol. 186, pp. 255-275, 1993.

[4] C. H. Guo and P. Lancaster, "Iterative solution of two matrix equations," Mathematics of Computation, vol. 68, no. 228, pp. 1589-1603, 1999.

[5] I. G. Ivanov, V. I. Hasanov, and B. V. Minchev, "On matrix equations $X \pm A^{T} X^{-2} A=I$," Linear Algebra and its Applications, vol. 326, no. 1-3, pp. 27-44, 2001.

[6] B. Meini, "Efficient computation of the extreme solutions of $X+A^{*} X^{-1} A=Q$ and $X-A^{*} X^{-1} A=Q$," Mathematics of Computation, vol. 71, no. 239, pp. 1189-1204, 2002.

[7] Z. Y. Peng, S. M. El-Sayed, and X. L. Zhang, "Iterative methods for the extremal positive definite solution of the matrix equation $X+A^{*} X^{-\alpha} A=Q$," Journal of Computational and Applied Mathematics, vol. 200, no. 2, pp. 520-527, 2007.

[8] M. A. Ramadan, "Necessary and sufficient conditions for the existence of positive definite solutions of the matrix equation $X+A^{T} X^{-2} A=I$, International Journal of Computer Mathematics, vol. 82, no. 7, pp. 865-870, 2005.

[9] M. A. Ramadan, "On the existence of extremal positive definite solution of the matrix equation $X+A^{T} \sqrt{X^{-1}} A=I$," International Journal of Nonlinear Sciences and Numerical Simulation, vol. 6, no. 2, pp. 115-126, 2005.

[10] M. A. Ramadan and N. M. El-Shazly, "On the matrix equation $X+A \sqrt[2 m]{X^{-1}} A=I$," Applied Mathematics and Computation, vol. 173, no. 2, pp. 992-1013, 2006. 
[11] A. M. Sarhan, N. M. El-Shazly, and E. M. Shehata, "On the existence of extremal positive definite solutions of the nonlinear matrix equation $X^{r}+\sum_{i=1}^{m} A_{i}^{*} X^{\delta_{i}} A_{i}=I$," Mathematical and Computer Modelling, vol. 51, no. 9-10, pp. 1107-1117, 2010.

[12] S.-F. Xu, "Perturbation analysis of the maximal solution of the matrix equation $X+A^{*} X^{-1} A=P$, Linear Algebra and its Applications, vol. 336, pp. 61-70, 2001.

[13] X. Zhan and J. Xie, "On the matrix equation $X+A^{T} X^{-1} A=I$," Linear Algebra and its Applications, vol. 247, pp. 337-345, 1996.

[14] J. C. Engwerda, "On the existence of a positive definite solution of the matrix equation $X+A^{T} X^{-1} A=I$, "Linear Algebra and its Applications, vol. 194, pp. 91-108, 1993.

[15] G. H. Golub and C. F. van Loan, Matrix Computations, Johns Hopkins University Press, Baltimore, Md, USA, 1989.

[16] G. W. Stewart and J. G. Sun, Matrix Perturbation Theory, Academic Press, Boston, Mass, USA, 1990. 


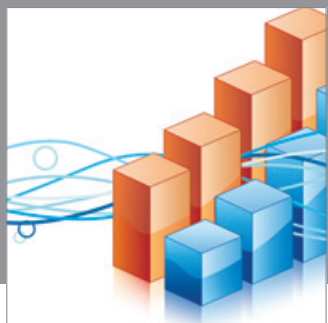

Advances in

Operations Research

mansans

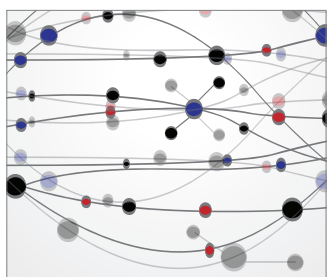

The Scientific World Journal
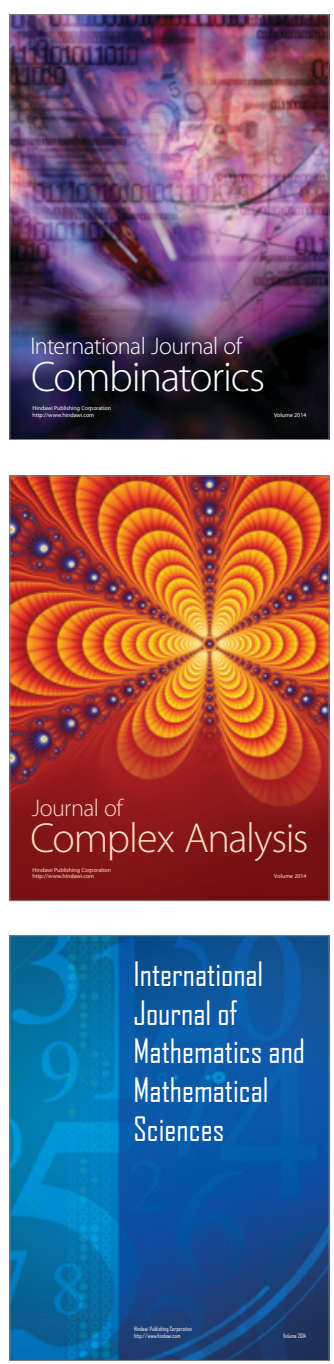
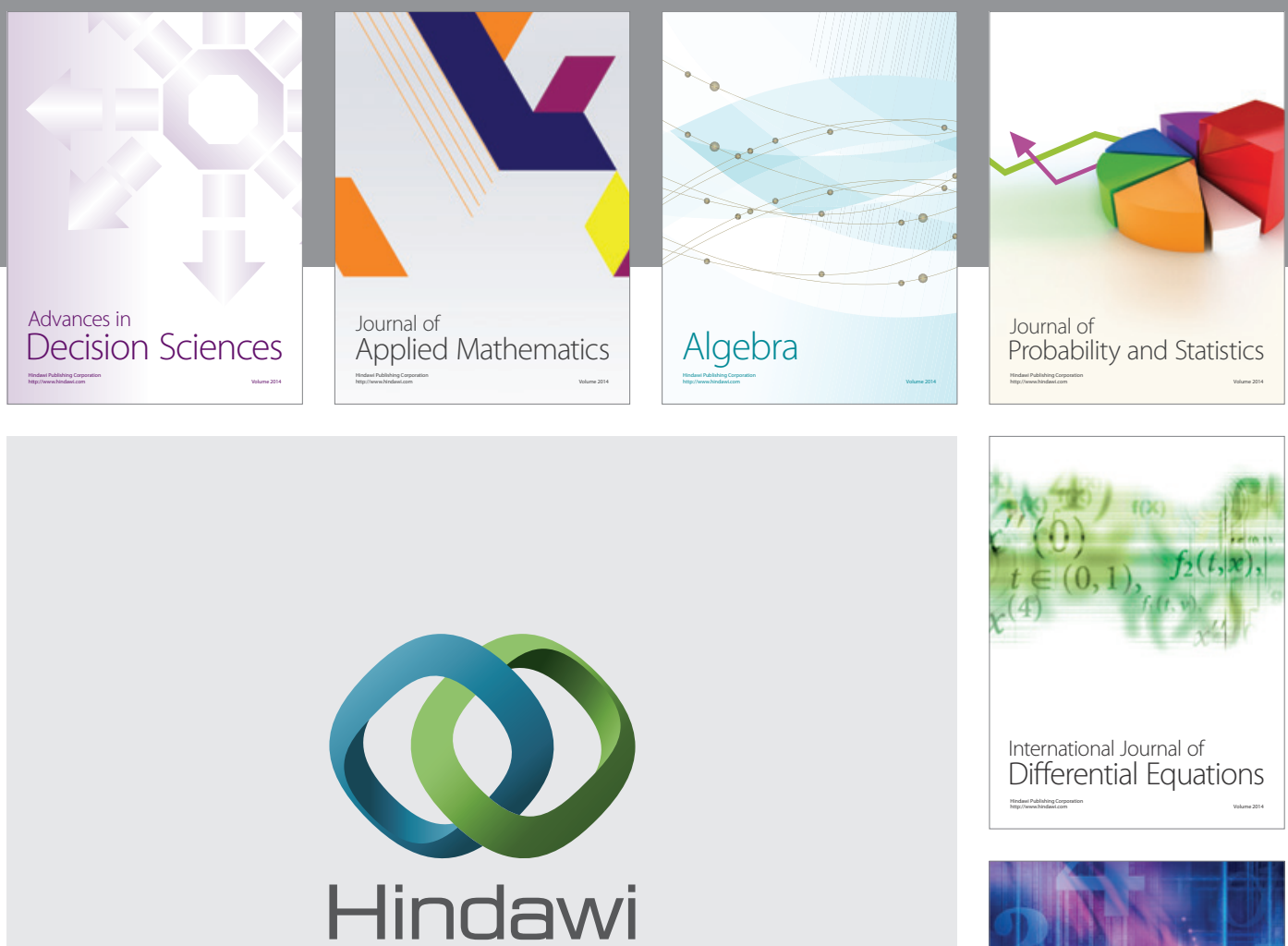

Submit your manuscripts at http://www.hindawi.com
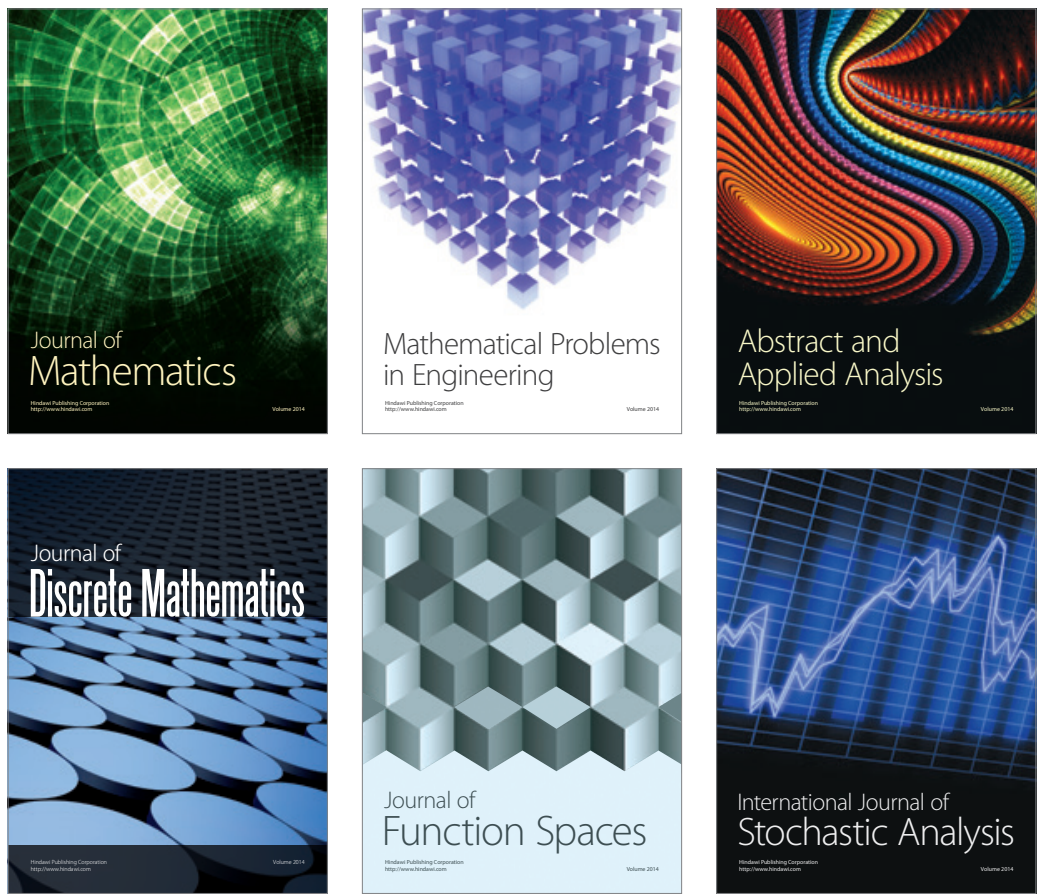

Journal of

Function Spaces

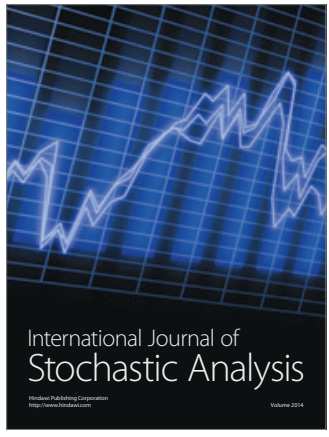

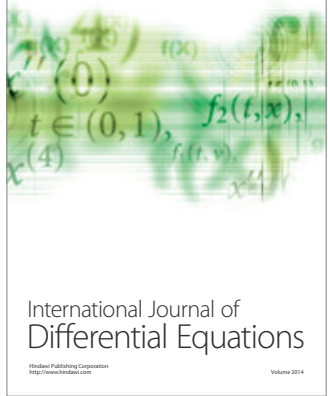
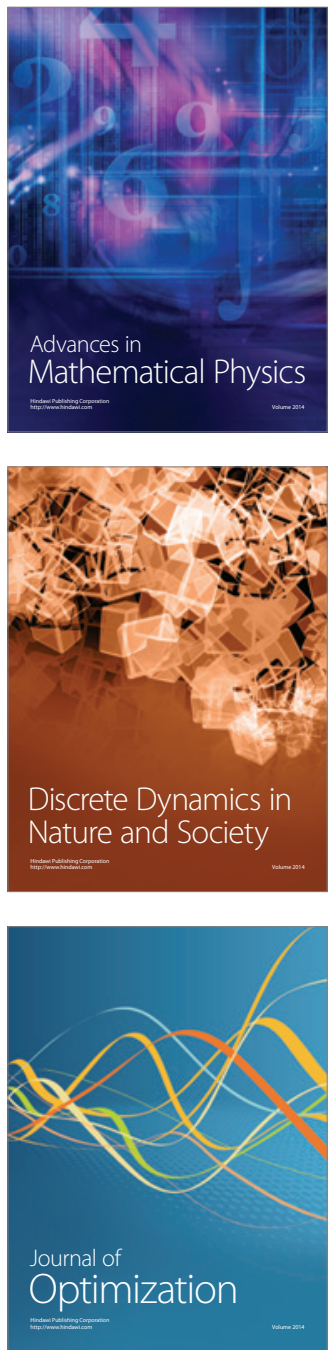